\title{
Lebensqualität bei Jugendlichen mit Vocal Cord Dysfunction
}

\author{
Quality of Life for Adolescents with Vocal Cord Dysfunction
}

Autoren

Institute
J. Schulze ${ }^{1}$, S. Weber ${ }^{1, *}$, S. Oddo ${ }^{2}$, M. Rosewich', M. A. Rose ${ }^{1}$, S. Zielen ${ }^{1}$

${ }^{1}$ Pädiatrische Pneumologie, Allergologie und Mukoviszidose, Zentrum für Kinder- und Jugendmedizin, Klinikum der J. W. Goethe-Universität, Frankfurt am Main

${ }^{2}$ Klinik für Psychiatrie, Psychosomatik und Psychotherapie des Kindes- und Jugendalters, Zentrum für Kinder- und Jugendmedizin und Klinik für Geburtshilfe und Pränatalmedizin, Klinikum der J. W. Goethe-Universität, Frankfurt am Main eingereicht 7.6. 2012 akzeptiert nach Revision 9. 7. 2012

\section{Bibliografie}

Dol http://dx.doi.org/ 10.1055/s-0032-1310105 Online-Publikation: 7.8.2012 Pneumologie 2012; 66: 596-601 (c) Georg Thieme Verlag KG Stuttgart · New York ISSN 0934-8387

Federführender Autor Dr. med. Johannes Schulze Pädiatrische Pneumologie, Allergologie und Mukoviszidose Zentrum für Kinder- und Jugendmedizin Klinikum der J. W. GoetheUniversität Frankfurt am Main Theodor Stern Kai 7 60590 Frankfurt am Main johannes.schulze@kgu.de

\section{Zusammenfassung \\ V}

Hintergrund: Die Vocal Cord Dysfunction (VCD) zählt zu den funktionellen Atemwegserkrankungen. Eine psychosomatische Genese wird diskutiert, und über Assoziationen mit Befunden wie Depression, Angst und sozialem Stress wird berichtet. Ziel ist die Untersuchung von Verhaltensauffälligkeiten und emotionalen Beschwerden von jugendlichen Patienten mithilfe von standardisierten Verhaltensfragebögen.

Methode: Es wurden 31 Patienten (8-16 Jahre) mit dem klinischen Verdacht einer VCD mithilfe der Verhaltensfragebögen Youth-Self-Report (YSR/11 - 18) sowie der Einschätzung der Eltern anhand der analogen Child-Behavior-Checklist (CBCL/6-18) untersucht. Im ersten Abschnitt werden Aktivitäten, soziale Kompetenzen und schulische Leistungen erfasst. Der zweite Teil umfasst verschiedene Syndromskalen, die emotionale Auffälligkeiten wie Depressivität oder Ängstlichkeit, Verhaltensauffälligkeiten wie Dissozialität und Aggressivität, soziale Probleme und körperliche Beschwerden erfassen.

Ergebnisse: Im Durchschnitt ergaben sich für VCD-Patienten keine signifikanten Unterschiede zu Referenzpopulationen. Prozentual gesehen, zeigten jedoch die Syndromskalen beider Fragebögen deutlich stärkere Tendenzen zu psychischen Auffälligkeiten (YSR 16,7\%, CBCL 20\%) im Vergleich zur Norm (2\%). Nach Angaben der Jugendlichen bestehen vor allem internale Auffälligkeiten (sozialer Rückzug, körperliche Beschwerden, Angst/Depressivität). Die Eltern berichten häufiger als die Jugendlichen über „körperliche Beschwerden“ (13,3\%) und „aggressives Verhalten“ (10\%).

Schlussfolgerungen: Wir fanden bei Patienten mit VCD Tendenzen psychischer Auffälligkeiten, die v.a. Depressivität, Ängstlichkeit und sozialen Rückzug umfassen. Weitere Untersuchungen sollten das Augenmerk auf derartige Faktoren richten

\section{Abstract \\ $\nabla$}

Background: Vocal cord dysfunction (VCD) is a functional breathing disorder. A psychosomatic aetiology has been discussed and associations with depression, anxiety disorders, and social stress have been reported. We have undertaken a screening of behavioural and emotional problems in adolescent patients using standardised questionnaires.

Methods: Thirty-one patients ( $8-16$ years) with the clinical suspicion of VCD were investigated using the Youth-Self-Report (YSR/11-18) and for the assessment of the parents we used the analoguous Child-Behaviour-Checklist (CBCL/6-18). YSR and CBCL contain two sub-areas: (a) competence scales that measure the child's participation in activities, social skills and school achievements and (b) items that contain subscales for emotional problems such as depressive and anxiety symptoms, conduct problems such as oppositional defiant problems and aggressive behaviour, social problems and physical complaints.

Results: On average, the features of VCD patients were not significantly different from those of the reference population. But we did observe tendencies of psychological problems (YSR 16.7\%, CBCL $20 \%$ ) compared with the standard (2\%) in the syndrome scales of both questionnaires Adolescents reported particularly more internalising disorders such as social retreat, physical complaint and anxiety and depressive symptoms. The parents reported more often "physical complaints" (13.3\%) and "aggressive behaviour" (10\%).

Conclusions: We found tendencies of psychological strain, mainly social retreat, physical complaints and anxiety and depressive symptoms. Further investigations should focus on those

\footnotetext{
* Lebensqualität bei Jugendlichen mit Vocal Cord Dysfunction, 33. Jahrestagung der Gesellschaft für Pädiatrische Pneumologie (GPP) Graz, 31. März-2.April 2011.
} 
aber auch psychosomatisch bedingte körperliche Beschwerden weiter untersuchen. Persönlichkeit und Belastungssituationen der Eltern sollten mit erfasst werden, um die hier gefundenen Angaben der Eltern bezüglich erhöhter Aggressivität und körperlicher Beschwerden ihrer VCD-Kinder vor dem Hintergrund der eigenen psychischen Belastung valider beurteilen zu können. Bei den auffälligen Familien empfehlen wir das Angebot von Familientherapien, Familiengesprächen oder ein Elterncoaching.

\section{Einleitung}

Patienten mit funktionellen Atemstörungen des Larynx, insbesondere Patienten mit Vocal Cord Dysfunction (VCD), leiden unter anfallsartiger Atemnot, Engegefühl im Hals und beschreiben auffällige Atemgeräusche [1]. Obwohl es zahlreiche Publikationen zur VCD gibt, kommt es häufig zu Fehldiagnosen und zur Verwechslung mit akuten Asthma-Anfällen. Neben der schwierigen Diagnostik wird eine psychische Genese der VCD diskutiert. Es gibt eine Reihe von Arbeiten, die die Atemstörungen mit psychologischen Auffälligkeiten in Verbindung bringen. Bei Jugendlichen hatten in der Untersuchung von Landwehr et al. [2] sechs von sieben Patienten mit VCD eine psychiatrische Diagnose. In einem anderen Kollektiv lag bei 55\% der Patienten sozialer Stress, verbunden mit Hochschulsport, vor [3]. Die VCD wird als Konversionsstörung im Jugendlichenalter betrachtet [4] sowie als eine Somatisierung von unterdrücktem Ärger und Depression [5]. Ein Zusammenhang mit sexuellem Missbrauch ist beschrieben [6]. Eine Autorengruppe aus Denver untersuchte einen Zusammenhang zwischen VCD und psychologischen Auffälligkeiten bei Jugendlichen mit VCD [7] systematisch mittels des „youth selfreport“ (YSR) [8] und der „child behavior checklist“ (CBCL) [9]. Insgesamt gibt es Evidenz dafür, dass Patienten mit VCD ein erhöhtes Risiko für psychiatrische und familienpsychologische Funktionsstörungen haben, jedoch ist die Aussagekraft der relevanten Literatur durch methodische Schwächen limitiert [7].

Um für den deutschsprachigen Raum systematisch psychische Auffälligkeiten bei Jugendlichen mit anfallsartiger Atemnot zu untersuchen, erhielten Patienten mit Verdacht auf funktionelle Atembeschwerden und deren Eltern den YSR bzw. den CBCL Fragebogen. Die Ergebnisse der Untersuchung wurden mit der Repräsentativstichprobe der Tests verglichen.

\section{Material und Methoden \\ $\nabla$}

\section{Patienten und VCD-Diagnostik}

In die offene Studie wurden 31 Patienten im Alter von 8 -16 Jahren (28 weiblich, drei männlich) eingeschlossen, die sich in unserer Ambulanz für Pneumologie und Allergologie im Zeitraum von Februar 2009 bis Januar 2011 mit anfallsartiger Atemnot vorstellten ( Tab.1). Der Verdacht einer VCD wurde anhand von drei Kriterien gestellt: Atemnot bei körperlicher Belastung, Engegefühl im Halsbereich und wenn die Patienten nicht - wie zu erwarten - auf eine etablierte Asthmatherapie ansprachen. Die Patienten hatten keinen chronischen Husten und keine Erkrankung der oberen Atemwege, wie eine chronische Rhinitis oder Sinusitis.

Zunächst wurden die Patientendaten erhoben, und Kinder und Eltern füllten die Fragebögen aus. Im Anschluss wurde ein Lungenfunktionstest durchgeführt (MasterScreen, CareFusion Ltd., Hoechberg, Deutschland), und es erfolgte eine direkte Laryngoskopie (Video ENF-V2 Laryngoskop, Olympus ${ }^{\circledR}$ ). Bei nor- emotional problems as well as on psychosomatically caused physical problems. Personality and psychological stress of the parents should be included in the investigation in order to evaluate the reports of the parents on higher aggressive behaviour and enhanced physical problems of their children in relation to their own psychological strain. We suggest family therapies, family counselling, or parental coaching as a therapeutic approach.

maler Basis-Lungenfunktion führten wir eine MethacholinProvokation mit steigenden Dosen von Methacholin durch, bis zum Abfall der Einsekundenkapazität um 20 Prozent, im Vergleich zum Ausgangswert, oder bis die Höchstdosis des Methacholin erreicht war [10]. Nach der Methacholin-Provokation erfolgte eine zweite direkte Laryngoskopie [11].

Die Untersuchung wurde von der Ethikkommission der J. W. Goethe-Universität, Frankfurt am Main, bewilligt. Alle Studienteilnehmer - und bei Minderjährigen deren Eltern - gaben zum Anfang ihr schriftliches Einverständnis. Die Studie wurde registriert unter Clinical trials gov: NCT 00906867.

\section{Fragebögen}

Unsere Untersuchung zu psychischen Auffälligkeiten stützte sich auf zwei Fragebögen, den Youth-Self-Report (YSR) für Jugendliche im Alter von 11 bis 18 Jahren [8] und die Child-BehaviorChecklist (CBCL) [9] für Kinder und Jugendliche im Alter von sechs bis 18 Jahren. Der YSR ist von den Jugendlichen selbst, die CBCL ist von den Eltern auszufüllen. Sie dienen der Selbst- bzw. Fremdeinschätzung, und beide Fragebögen sind nahezu identisch. Im ersten Teil werden psychosoziale Kompetenzen des Jugendlichen erfragt, der zweite Teil besteht aus 120 Items, in denen Verhaltensauffälligkeiten, emotionale Auffälligkeiten und körperlicher Beschwerden beschrieben werden. Der Beurteilungszeitraum umfasst die letzten sechs Monate. Die Beurteilung erfolgt anhand einer dreistufigen Likert-Skala von $0=$ „nicht zutreffend“ über 1 = „etwas oder manchmal zutreffend“ bis 2 = „genau oder häufig zutreffend“. Aus den Items des zweiten Teils des Fragebogens werden acht Syndromskalen gebildet. 33 Items sind keiner Skala zugeordnet und gehen zusammen mit den anderen Items in den 118 Items umfassenden Gesamtauffälligkeitswert ein [12].

$\mathrm{Zu}$ den internalisierenden Syndromskalen gehören beispielsweise „Sozialer Rückzug“ mit den Items „alleine, schüchtern, verschlossen und häufiger traurig verstimmt sein“ und „ängstlich/ depressiv“ mit den Items „Ängstlichkeit, Nervosität, Einsamkeit, soziale Ablehnung, Minderwertigkeitsgefühle und traurige Verstimmung“. Zu den externalisierenden Syndromskalen gehören „Dissoziales Verhalten“ mit den Items „Lügen, Stehlen, Fluchen

Tab. 1 Patientencharakteristika.

\begin{tabular}{|lll|} 
& & Gesamt \\
\hline Patienten & $(\mathrm{n})$ & 31 \\
\hline weiblich/männlich & $(\mathrm{n})$ & $28 / 3$ \\
\hline $\mathrm{VCD} /$ BHR & $(\mathrm{n})$ & $14 / 17$ \\
\hline erste Symptome & $(\mathrm{J} a \mathrm{hre}, \mathrm{MW} \pm \mathrm{SA})$ & $11,9 \pm 2,7$ \\
\hline Alter bei Diagnose & $(\mathrm{J} a \mathrm{hre}, \mathrm{MW} \pm \mathrm{SA})$ & $12,8 \pm 2,3$ \\
\hline Diagnose Asthma & $(\mathrm{n}, \%)$ & $10(32,3)$ \\
\hline Asthmatherapie & $(\mathrm{n}, \%)$ & $20(64,5)$ \\
\hline
\end{tabular}

Die Patienten waren überwiegend weiblich. Zwischen den ersten Symptomen der Atemnot und der Diagnosestellung verging im Schnitt ein Jahr. Bei Erstvorstellung hatten $32 \%$ die Diagnose Asthma und $65 \%$ eine Asthmatherapie. 
Tab. 2 YSR-Fragebogen $n=24$

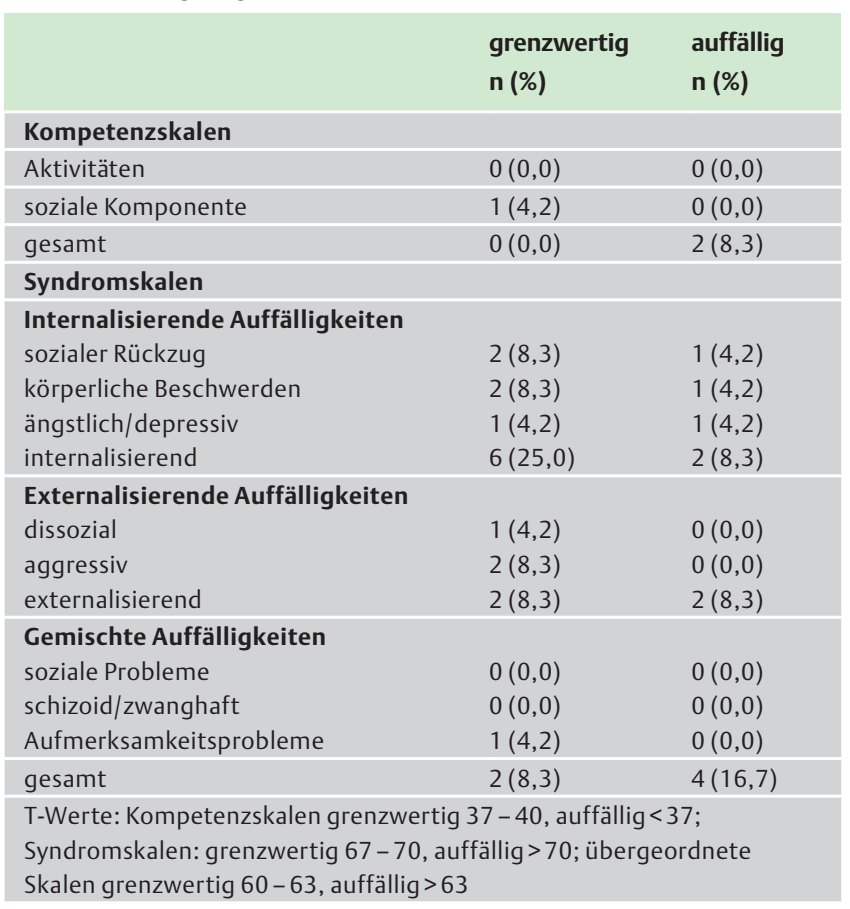

Ergebnisse des Fragebogens für Jugendliche (Youth-Self-Report).

Eine Abweichung von der Norm (2\%) besteht in der Gesamtskala und in den internalisierenden Skalen.

und von zuhause Weglaufen“ und „Aggressives Verhalten“ mit den Items „Wutausbrüche, Eifersucht und Affektlabilität“.

Bei der Auswertung der Fragebögen ergeben sich Summenwerte, die entsprechenden T-Werten und Prozenträngen zugeordnet werden können.

Die deutsche Normierung der Ergebnisse erfolgte 1994 anhand der PAK-KID-Studie [12]. Diese ergab, dass die in der Befragung auffälligsten zwei Prozent (2\%) der Repräsentativstichprobe klinisch ebenfalls als auffällig beurteilt werden können. Daraus ergibt sich ein signifikantes Ergebnis für die Kompetenzen ab einem T-Wert kleiner 41. Der alle Stichpunkte umfassende Gesamtwert für die Syndromskala wird ab einem T-Wert größer 59 als „grenzwertig“ und größer 63 als „auffällig“ interpretiert.

Auffällige Ergebnisse in den Fragebögen liefern keine psychiatrischen Diagnosen, sondern zeigen lediglich Tendenzen zu Verhaltensauffälligkeiten auf, die einer genaueren psychiatrischen Abklärung bedürfen.

\section{Statistik}

Nach Prüfung auf Normalverteilung wurde anhand des Student tTests untersucht, ob sich die statistischen Mittelwerte der vorliegenden Patienten von der Normstichprobe unterscheiden. Der Zusammenhang zweier Stichproben wurde mithilfe der PearsonKorrelation oder der Rangkorrelation nach Spearman berechnet. Ein $\mathrm{p}<0,5$ wurde als statistisch signifikant angesehen.

\section{Ergebnisse}

$\nabla$

Von den 31 Patienten berichteten 29 über Atemnot bei körperlicher Belastung, 27 hatten ein Engegefühl im Hals, und 16 von 20 Patienten $(80 \%)$ reagierten nicht, wie zu erwarten, auf eine Asthmatherapie. Mit der Methode der direkten Laryngoskopie vor
Tab. 3 CBCL-Fragebogen $n=30$.

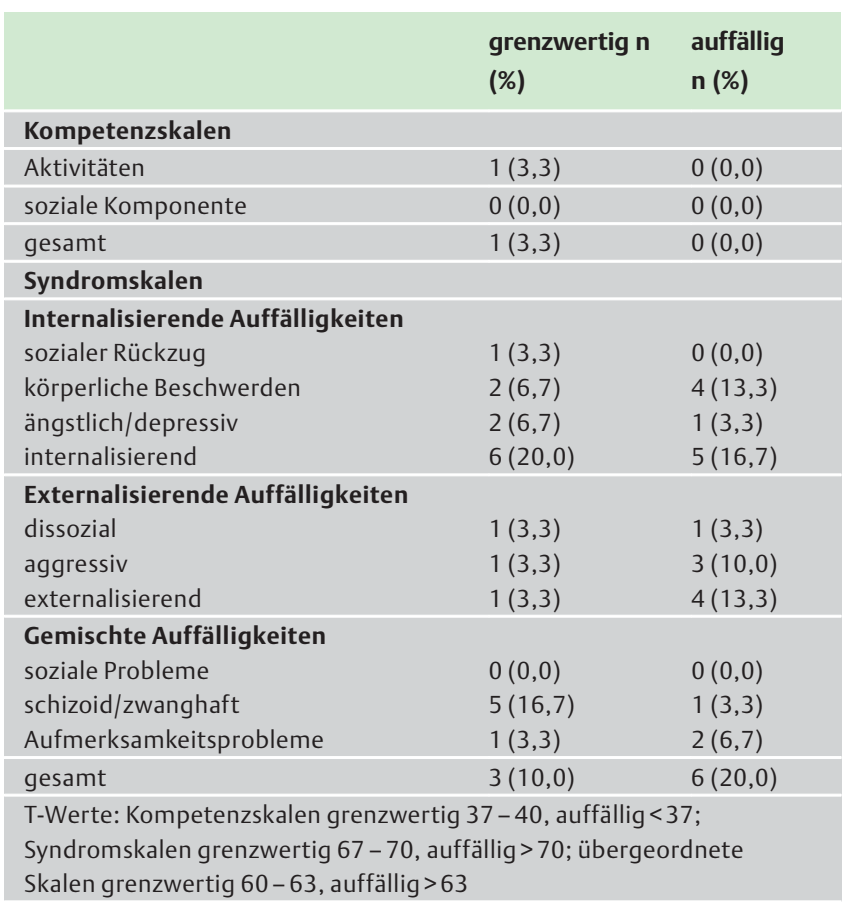

Ergebnisse des Fragebogens für die Eltern (Child-Behaviour-Checklist). Eine Abweichung von der Norm ( $2 \%$ ) besteht in der Gesamtskala, bei den körperlichen Beschwerden und dem aggressiven Verhalten.

und nach Methacholin-Provokation konnten wir bei 14 Patienten eine VCD und bei 17 Patienten eine bronchiale Überempfindlichkeit (BHR) diagnostizieren [11].

Von den 31 Kindern und Jugendlichen konnten insgesamt 24 YSR-Verhaltensfragebögen ausgewertet werden. Fünf Patienten lagen unterhalb der Altersnorm, und zwei Patienten hatten den Fragebogen lückenhaft ausgefüllt.

Acht der 24 Patienten (33,3\%) waren internalisierend grenzwertig bis auffällig. Im Bereich der internalisierenden Auffälligkeiten waren alle drei Bereiche, sozialer Rückzug, körperliche Beschwerden sowie ängstlich/depressiv, in etwa gleichem Maße betroffen. Vier (16,6\%) der Befragten erzielten ein grenzwertiges bis auffälliges Ergebnis in der Gesamtbetrachtung der externalisierenden Verhaltensweisen. Der Gesamtwert aller Items war bei zwei $(8,3$ $\%)$ Patienten im grenzwertigen und bei vier (16,7\%) Patienten im auffälligen Bereich. Im Bereich der Kompetenzen zeigten nur zwei $(8,3 \%)$ der Patienten Auffälligkeiten ( $\checkmark$ Tab.2). Für beide Gruppen, VCD und BHR, fanden sich keine Unterschiede, so hatten von den vier auffälligen Patienten im Gesamtwert zwei eine VCD und zwei eine BHR.

Die CBCL wurde für 30 Patienten ausreichend beantwortet, bei einem Patienten war der Fragebogen lückenhaft ausgefüllt. Der höchste Wert ergab sich für die internalisierenden Auffälligkeiten, 36,7\% der Eltern beurteilten die Kinder mit grenzwertigen und auffälligen Werten. Hier fanden sich deutlich erhöhte Werte bei den körperlichen Beschwerden (20\%) und weniger häufig bei ängstlich/depressivem Verhalten (10\%). Externalisierende Auffälligkeiten (überwiegend Aggressivität) beschrieben 16,6\% der Eltern. Der Gesamtwert für alle Stichpunkte war in 10\% der Beurteilungen im grenzwertigen und in $20 \%$ der Beurteilungen im auffälligen Bereich. Es zeigten sich wenig Auffälligkeiten bei den Kompetenzen (3,3\%). ( Tab.3). Für beide Gruppen, VCD und BHR, fanden sich keine Unterschiede, so hatten von den sechs 
Tab. 4 Vergleich T-Werte Kinder und Eltern, $\mathrm{n}=24$.

\begin{tabular}{|c|c|c|c|c|c|c|}
\hline & & Kinder & Eltern & $\mathbf{p}$ & Pearson-Korrelation & $\mathbf{p}$ \\
\hline Kompetenzen & $\mathrm{MW} \pm \mathrm{SA}$ & $53,2 \pm 7,8$ & $55,6 \pm 7,9$ & 0,22 & $r=0,28$ & 0,19 \\
\hline internalisierend & $\mathrm{MW} \pm \mathrm{SA}$ & $51,6 \pm 12,5$ & $54,8 \pm 9,7$ & 0,33 & $r=0,44$ & 0,03 \\
\hline externalisierend & $\mathrm{MW} \pm \mathrm{SA}$ & $50,8 \pm 9,8$ & $51,5 \pm 10,2$ & 0,79 & $r=0,63$ & 0,001 \\
\hline gesamt & $\mathrm{MW} \pm \mathrm{SA}$ & $51,3 \pm 11,1$ & $53,9 \pm 10,4$ & 0,41 & $r=0,54$ & 0,006 \\
\hline
\end{tabular}

Die T-Werte für Jugendliche und Eltern unterscheiden sich nicht (Student t-Test). Für die in- und externalisierenden Skalen sowie die Gesamtskala gibt es eine signifikante Korrelation.

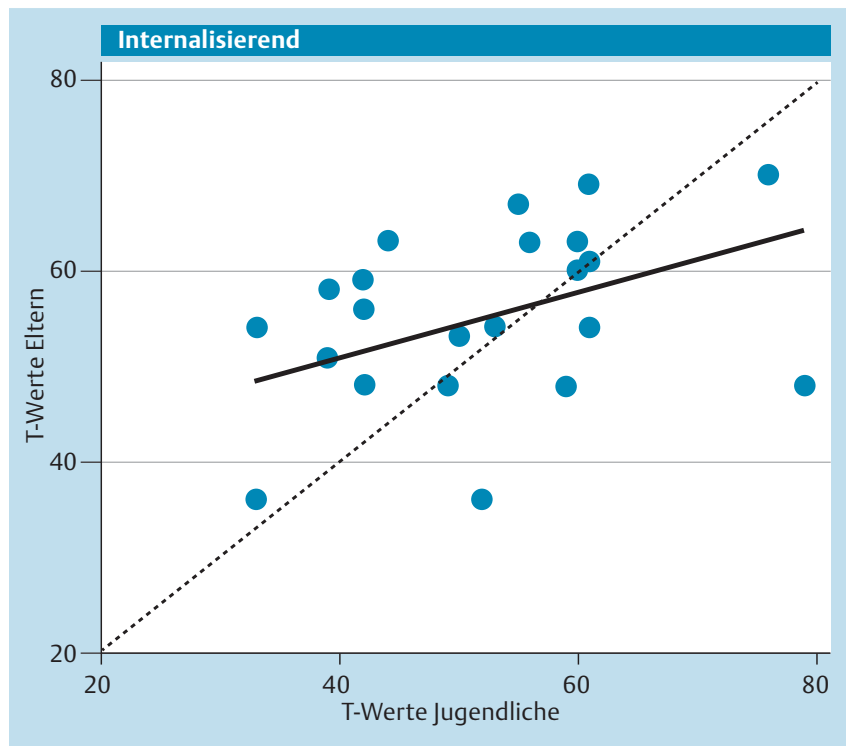

Abb. 1 Korrelation der internalisierenden T-Werte im Vergleich Jugendliche und Eltern $(r=0,44, p=0,03)$.

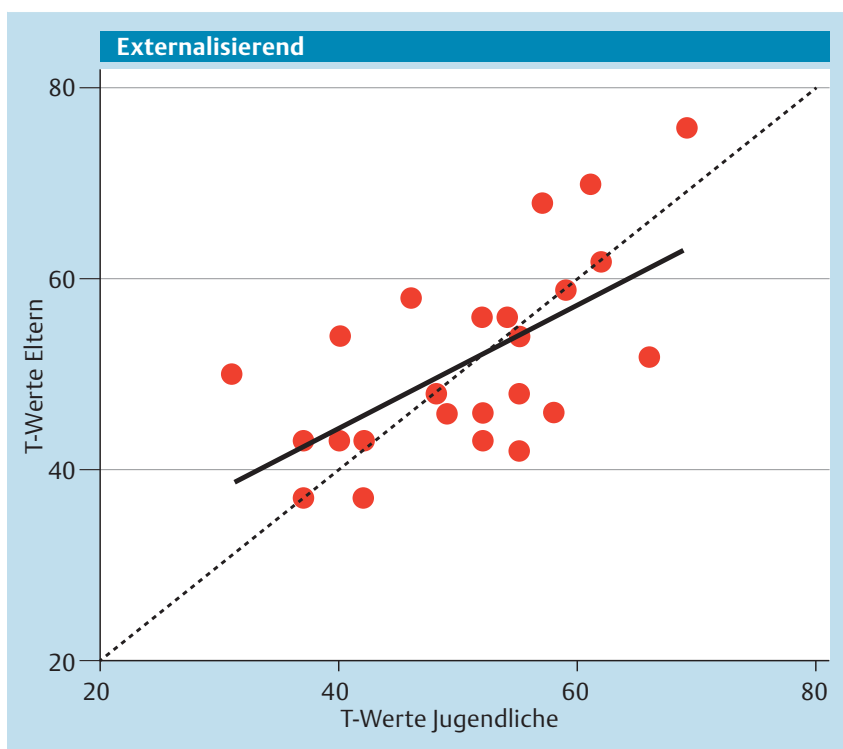

Abb. 2 Korrelation der externalisierenden T-Werte im Vergleich Jugendliche und Eltern $(r=0,63, p=0,001)$.

\section{Diskussion}

Dies ist die erste Untersuchung in Deutschland, die mithilfe von standardisierten Fragebögen psychische Auffälligkeiten bei Jugendlichen untersucht, die sich mit Symptomen der Vocal Cord Dysfunction in einer spezialisierten Ambulanz vorstellen.

Die Patienten und ihre Eltern erhielten zuerst die Fragebögen, dann erfolgte die Diagnostik der VCD mithilfe der Laryngoskopie und Methacholin-Provokation. Dabei wurde bei 14 Patienten die Diagnose VCD und bei 17 Patienten die Diagnose BHR gestellt. Patienten mit VCD haben eine akute wiederkehrende Atemnot mit einem Engegefühl im Hals [1]. Bei Jugendlichen wird die VCD typischerweise durch körperliche Anstrengung getriggert [13], und die Symptome reagieren nicht auf eine Asthmatherapie mit ß-Mimetika [14]. Für fast alle Patienten in unserer Untersuchung treffen diese Anzeichen der VCD zu, und anhand der klinischen Beurteilung konnten beide Patientengruppen nicht unterschieden werden. Das heißt, es gibt eine Gruppe von jugendlichen und überwiegend weiblichen Patienten mit BHR, die wie eine VCD imponiert. Erstaunlicherweise finden sich psychische Auffälligkeiten, gleich verteilt, in beiden Gruppen. Aufgrund der zu geringen Fallzahl konnten wir bei der Auswertung der Fragebögen nicht zwischen der VCD- und der BHR-Gruppe unterscheiden.

Die Übereinstimmung der T-Werte zwischen Selbstbeurteilung der Jugendlichen (YSR) und der Fremdeinschätzung der Eltern (CBCL-Fragebögen) weist darauf hin, dass die Jugendlichen „nicht sozial erwünscht" angekreuzt haben. 
Die Auswertung der YSR-Fragebögen ergibt auf den ersten Blick wenig Auffälligkeiten, doch haben die Jugendlichen erhöhte Werte in der Gesamtskala. Im Jahr 1999 wurden 508 Kinder, Jugendliche und deren Eltern, die eine hausärztliche Praxis in Aachen aufsuchten, mithilfe des YSR und CBCL befragt [15]. Hier finden sich für die Jugendlichen ähnliche Werte in der Gesamtskala. Doch sind diese zu gleichen Teilen auf internalisierende und externalisierende Auffälligkeiten zurückzuführen. In unserer Untersuchung ergibt sich der erhöhte Gesamtscore überwiegend aus grenzwertigen und auffälligen Befunden in der internalisierenden Syndromskala.

In der Aachener Untersuchung hatten 12,5 bis $27 \%$ der Jugendlichen Verhaltensauffälligkeiten, Leistungs- oder emotionale Störungen [15]. Dabei zeigten 13,3\% der Jungen und 14,0\% der Mädchen Auffälligkeiten in der internalisierenden Syndromskala. In unserer Untersuchung gibt es eine deutliche Tendenz zu internalisierendem Verhalten, die möglicherweise aufgrund der kleinen Stichprobe nicht eindeutiger ausfällt. Patienten mit VCD reagieren auf anfallsartige Atemnot typischerweise mit Panik und Erstickungsangst und sind sehr beunruhigt über das mögliche Wiederauftreten der Störung [14]. Die Erhöhung der Werte unserer Gruppe weist auf eine soziale Rückzugstendenz mit ängstlich depressivem Verhalten hin.

Dabei gibt es eine deutliche Diskrepanz zwischen Elternurteil und Selbsteinschätzung. Die Eltern berichten prozentual häufiger über körperliche Beschwerden und externalisierende Auffälligkeiten, v.a. aggressives Verhalten der Kinder. In der Regel liegt das Selbsturteil von Kindern und Jugendlichen im Alter von 11 bis 18 Jahren auf allen Skalen über der Einschätzung der Eltern [12], d.h. die Jugendlichen beurteilen sich selbst als auffälliger hinsichtlich Ängstlichkeit, Depressivität und Störungen des Sozialverhaltens. Für die höheren Ausprägungen im Urteil der Jugendlichen sind vermutlich vor allem die unterschiedliche Informationsbasis von Eltern und Jugendlichen und situationsspezifisch unterschiedliches Verhalten Jugendlicher verantwortlich. Eltern haben hinsichtlich mancher Lebensbereiche von Jugendlichen keinen ausreichenden Einblick (z.B. Gleichaltrigengruppe, Schule) und nehmen deshalb bestimmte Verhaltensweisen gar nicht wahr [12]. In der Studie aus Denver [7] erhielten die Jugendlichen mit VCD in der Beurteilung der Eltern in den internalisierenden Skalen ebenfalls signifikant von der Norm abweichende T-Werte. In der Untersuchung wurden Jugendliche und Eltern zusätzlich in einem Untersucher-basierten diagnostischen Interview untersucht (CAPA, child and adolescent psychiatric assessment). Im Vergleich zu einer Kontrollgruppe hatten Patienten mit VCD eine größere Anzahl von psychiatrischen Diagnosen, vor allem Ängste wie Trennungsangst, Überängstlichkeit und Agarophobie. Zum größeren Teil bestanden die Angstsymptome jedoch schon, bevor die Atemwegssymptome auftraten.

Auf der anderen Seite ist die typische Reaktion auf die akute Atemnot Panik und Erstickungsangst [13]. Da wir die Fragebögen erhoben, nachdem die Patienten schon erkrankt waren und wir keine weiterführenden Interviews führten, können wir die Frage nicht klären, ob es sich bei den Angststörungen um eine primäre, die Erkrankung auslösende oder begünstigende Störung handelt oder ob diese nicht auch sekundär Folge intermittierender bedrohlich erlebter Atemnotattacken sein können. Insgesamt besteht wenig Klarheit für den Stellenwert psychiatrischer Erkrankungen in der Pathophysiologie der VCD [14].

Bei habituellem Husten ist bekannt, dass dieser zu vermehrter Aufmerksamkeit in der Familie führen kann, aber auch, dass die Umstehenden mehr entnervt sind als der Patient selbst [14]. Bei der VCD sind nicht nur die Patienten, sondern auch die Umgebungspersonen über das mögliche Wiederauftreten der Atemnot sehr beunruhigt [14]. Die Situation belastet die Eltern offensichtlich, die Einschätzung des aggressiven Verhaltens ist möglicherweise ein Zeichen dafür, dass die Eltern mit den Jugendlichen oder der Situation überfordert sind. Gleiches Verhalten kann auch dann unterschiedlich beurteilt werden, wenn oppositionelle Verhaltensweisen von den Jugendlichen weniger ausgeprägt eingeschätzt werden als von den Eltern [12].

Zusätzlich gibt es Alterseffekte und Geschlechtsdifferenzen [12]. Nach Einschätzung der Eltern nehmen internalisierende Auffälligkeiten und dissoziale Verhaltensweisen mit dem Alter der Kinder eher zu. Bei Mädchen fanden sich für die internalen Störungen etwas höhere Werte, allerdings waren die Geschlechtsdifferenzen lediglich für die Skala „körperliche Beschwerden“ statistisch signifikant [12]. Aufgrund der kleinen Stichprobe konnten wir keine differenzierte Analyse durchführen. Jedoch war der überwiegende Anteil unserer Patienten weiblich. Bei aggressiven Verhaltensweisen werden von den Eltern über die gesamte Altersspanne hinweg Jungen im Vergleich zu Mädchen als auffälliger eingeschätzt [12].

Jugendliche mit VCD haben keine Schwierigkeiten bei ihren sportlichen Aktivitäten, den sozialen Kompetenzen und in der Schule, wie auch in anderen Studien nachgewiesen [7].

Es darf nicht übersehen werden, dass in unserer Untersuchung nur etwa $20 \%$ der Fragebögen auffällige Werte zeigten. Unstrittig ist, dass neben möglichen psychosomatischen Ursachen somatische Faktoren auslösend oder mit auslösend für die Entstehung einer VCD sein können. Es gibt zunehmend Veröffentlichungen über den Zusammenhang von gastro-ösophagealem Reflux (GÖR) und VCD. In einer Untersuchung hatten 18\% der Patienten mit VCD einen zugrundeliegenden Reflux [16]. Bei 19 von 22 Jugendlichen mit VCD fanden sich posteriore Veränderungen der Aryknorpel und ein Ödem zwischen den Aryknorpeln, wie sie typischerweise bei GÖR gesehen werden [17]. Wir hatten unsere Gruppe nicht systematisch für einen GÖR, z.B. mithilfe der 24h-pH-Metrie, untersucht. Es wurden einzig anamnestische Daten erhoben, hier hatten fünf der 31 Patienten Refluxsymptome. Eine weitere Ursache für eine VCD sind Infektionen der oberen Atemwege. Bei 17 von 27 Patienten mit VCD konnte ein Infekt der oberen Atemwege gefunden werden [18], und bei 213 Patienten mit VCD bestand bei 19\% ein „postnasal drip“ [19]. In einer anderen großen Serie mit 176 Patienten hatten $31 \%$ eine begleitende Rhinosinusitis [20]. In unserer Untersuchung hatten die Patienten keinen chronischen Husten und keine Erkrankung der oberen Atemwege, wie eine chronische Rhinitis oder Sinusitis. Wir fanden in der Untersuchung drei Patienten mit anatomischen Veränderungen: Vergrößerte Andenoide, eine überschießende Mukosa der Aryknorpel und der Verdacht einer Rekurrenzparese [11]. Dies zeigt, dass wir bei der laryngoskopischen Untersuchung mit der notwendigen Sorgfalt vorgegangen sind.

Für die Mehrzahl der Patienten sind die bekannten therapeutischen Optionen ausreichend. Dies ist üblicherweise eine Kombination von Schulung und Sprachtherapie $[13,14]$. Sehr hilfreich ist die Visualisierung der VCD/BHR während oder nach der laryngoskopischen Untersuchung ggf. mit Bio-Feedback. Die wirkungsvollste therapeutische Intervention ist die Sprachtherapie. Ziel ist eine Entspannung der Muskeln von Larynx, Hals, Schulter und Brustkorb mithilfe von Atem- und Entspannungsübungen [21-23]. Nach einer erfolgreichen Behandlung verschwinden die Symptome oft nicht ganz, jedoch sie sind weniger schwer 
und weniger häufig [13]. Zugrundeliegende Erkrankungen wie Infektionen oder ein GÖR sollten behandelt werden [14].

Im Falle, dass diese therapeutischen Maßnahmen keinen Erfolg haben, sollten die jugendlichen Patienten im Rahmen von psychosomatischen Anamneseuntersuchungen objektiv von examinierten Psychologen/Ärzten untersucht werden, um ein weiteres Maß zur Selbst- und Fremdeinschätzung der betroffenen Kinder und Eltern zu erhalten. Es sollten andere Screeninginstrumente parallel eingesetzt werden, da die hier angewandten Verfahren, streng genommen, nicht als psychiatrisches/psychosomatisches Screening im Sinne handfester psychischer Diagnosen fungieren, sondern eher einen Hinweis auf weitere psychosomatisch-psychiatrische Abklärung liefern können.

\section{Fazit}

In der Untersuchung von Jugendlichen mit den klinischen Zeichen einer VCD ist die Stichprobe sehr heterogen in der Wahrnehmung der Belastung, und die Eltern geben einen tendenziell höheren Leidensdruck an. Bei hohen Ausprägungen auf den Syndromskalen können Jugendliche als zum Teil psychisch und verhaltensauffällig eingeschätzt werden [24]. Insbesondere erhöhte körperliche Beschwerden, sozialer Rückzug sowie depressiv-ängstliche Symptome und aggressiv-dissoziales Verhalten wurden in der vorliegenden Studie erstmalig für deutsche Patienten mit VCD beschrieben.

Betroffenen Familien sollten Familientherapien, Familiengespräche oder ein Elterncoaching angeboten werden. Weitere Untersuchungen sollten eine spezifischere psychosomatischpsychiatrische Diagnostik vornehmen, mit einer Fokussierung auf internalisierende Faktoren (sozialer Rückzug, Depressivität, Ängstlichkeit) und körperliche Symptome. Persönlichkeit und Belastungssituationen der Eltern sollten mit erfasst werden.

\section{Interessenkonflikt}

\section{$\nabla$}

Die Autoren geben an, dass kein Interessenkonflikt besteht.

\section{Literatur}

1 Newman KB, Mason UG, Schmaling KB. Clinical features of vocal cord dysfunction. Am J Respir Crit Care Med 1995; 152: 1382 - 1386

2 Landwehr LP, Wood RP, Blager FB et al. Vocal cord dysfunction mimicking exercise-induced bronchospasm in adolescents. Pediatrics 1996; 98: $971-974$

3 Powell DM, Karanfilov BI, Beechler KB et al. Paradoxical vocal cord dysfunction in juveniles. Arch Otolaryngol Head Neck Surg 2000; 126: $29-34$
4 Geist R, Tallett SE. Diagnosis and management of psychogenic stridor caused by a conversion disorder. Pediatrics 1990; 86: 315-317

5 Kattan M, Ben-Zvi Z. Stridor caused by vocal cord malfunction associated with emotional factors. Clin Pediatr 1985; 24: 158-160

6 Tajchman UW, Gitterman B. Vocal cord dysfunction associated with sexual abuse. Clin Pediatr 1996; 35: 105-108

7 Gavin LA, Wamboldt M, Brugman $S$ et al. Psychological and family characteristics of adolescents with vocal cord dysfunction. J Asthma 1998; 35: $409-417$

8 Achenbach TM. Manual for the Youth Self-Report and 1991 Profile. Burlington, VT: Department of Psychiatry; University of Vermont; 1991

9 Achenbach TM. Manual for the Child Behavior Checklist/ 4-18 and 1991 Profile. Burlington, VT: Department of Psychiatry; University of Vermont; 1991

10 Schulze J, Rosewich M, Riemer C et al. Methacholine challenge - comparison of an ATS protocol to a new rapid single concentration technique. Respir Med 2009; 103: 1898 - 1903

11 Schulze J, Weber S, Rosewich $M$ et al. Vocal cord dysfunction in adolescents. Pediatr Pulmonol 2012; 47: 612-619

12 Döpfner M, PlückJ, Berner W et al. Psychische Auffälligkeiten von Kindern und Jugendlichen in Deutschland - Ergebnisse einer repräsentativen Studie: Methodik, Alter-, Geschlechts- und Beurteilereffekte. Z Kinder Jugendpsychiatr Psychother 1997; 25: 218-233

13 Tilles SA. Vocal cord dysfunction in children and adolescents. Curr Allergy Asthma Rep 2003; 3: 467-472

14 Grüber C, Lehmann C, Weiss $C$ et al. Somatoform respiratory disorders in children and adolescents-proposals for a practical approach to definition and classification. Pediatr Pulmonol 2012; 47: 199-205

15 Ziegert B, Neuss A, Herpertz-Dahlmann B et al. Psychische Auffälligkeiten von Kindern und Jugendlichen in der allgemeinärztlichen Praxis. Dtsch Arztebl 2002; 99: 1436 - 1441

16 Morris MJ, Allan PF, Perkins PJ. Vocal cord dysfunction: etiologies and treatment. Clin Pulm Med 2006; 13: 73-86

17 Powell DM, Karanfilov BI, Beechler KB et al. Paradoxical vocal cord dysfunction in juveniles. Arch Otolaryngol Head Neck Surg 2000; 126 : $29-34$

18 Andrianopoulos MV, Gallivan GJ, Gallivan KH. PVCM, PVCD, EPL, and irritable larynx syndrome: what are we talking about and how do we treat it? J Voice 2000; 14: 607-618

19 Brugman SM. The many faces of vocal cord dysfunction: what 36 years of literature tell us. Am J Respir Crit Care Med 2003; 167: A588

20 Perello MM, Gurevich J, Fitzpatrick T et al. Clinical characteristics of vocal cord dysfunction in two military tertiary care facilities. Am J Respir Crit Care Med 2003; 167: A788

21 Brugman SM, Simons SM. Vocal cord dysfunction: don't mistake it for asthma. Phys Sportsmed 1998; 26: 63-85

22 Sullivan MD, Heywood BM, Beukelman DR. A treatment for vocal cord dysfunction in female athletes: an outcome study. Laryngoscope 2001; 111: $1751-1755$

23 Wood RP, Milgrom H. Vocal cord dysfunction. J Allergy Clin Immunol 1996; 98: $481-485$

24 Döpfner M, Plück J, Bölte $S$ et al. Fragebogen für Jugendliche, Deutsche Bearbeitung des Youth Self-Report (YSR) der Child Behavior Checklist, Einführung und Anleitung zur Handauswertung. Köln: KJFD, Arbeitsgruppe Kinder-, Jugend- und Familiendiagnostik; 1998 